\title{
Integrated DNA methylation and copy-number profiling identify three clinically and biologically relevant groups of anaplastic glioma
}

Wiestler, B ; Capper, D ; Sill, M ; Jones, D T W ; Hovestadt, V ; Sturm, D ; Koelsche, C ; Bertoni, A ; Schweizer, L ; Korshunov, A ; Weiß, E K ; Schliesser, M G ; Radbruch, A ; Herold-Mende, C ; Roth, P ; Unterberg, A ; Hartmann, C ; Pietsch, T ; Reifenberger, G ; Lichter, P ; Radlwimmer, B ; Platten, M ; Pfister, S M ; von Deimling, A ; Weller, M ; Wick, W

\begin{abstract}
The outcome of patients with anaplastic gliomas varies considerably. Whether a molecular classification of anaplastic gliomas based on large-scale genomic or epigenomic analyses is superior to histopathology for reflecting distinct biological groups, predicting outcomes and guiding therapy decisions has yet to be determined. Epigenome-wide DNA methylation analysis, using a platform which also allows the detection of copy-number aberrations, was performed in a cohort of 228 patients with anaplastic gliomas (astrocytomas, oligoastrocytomas, and oligodendrogliomas), including 115 patients of the NOA-04 trial. We further compared these tumors with a group of 55 glioblastomas. Unsupervised clustering of DNA methylation patterns revealed two main groups correlated with IDH status: CpG island methylator phenotype (CIMP) positive $(77.5 \%)$ or negative $(22.5 \%)$. CIMP(pos) (IDH mutant) tumors showed a further separation based on copy-number status of chromosome arms 1p and 19q. CIMP(neg) (IDH wild type) tumors showed hallmark copy-number alterations of glioblastomas, and clustered together with CIMP(neg) glioblastomas without forming separate groups based on WHO grade. Notably, there was no molecular evidence for a distinct biological entity representing anaplastic oligoastrocytoma. Tumor classification based on CIMP and 1p/19q status was significantly associated with survival, allowing a better prediction of outcome than the current histopathological classification: patients with CIMP(pos) tumors with 1p/19q codeletion (CIMP-codel) had the best prognosis, followed by patients with CIMP(pos) tumors but intact 1p/19q status (CIMP-non-codel). Patients with CIMP(neg) anaplastic gliomas (GBM-like) had the worst prognosis. Collectively, our data suggest that anaplastic gliomas can be grouped by IDH and 1p/19q status into three molecular groups that show clear links to underlying biology and a significant association with clinical outcome in a prospective trial cohort.
\end{abstract}

DOI: https://doi.org/10.1007/s00401-014-1315-x

Posted at the Zurich Open Repository and Archive, University of Zurich

ZORA URL: https://doi.org/10.5167/uzh-98106

Journal Article

Accepted Version

Originally published at:

Wiestler, B; Capper, D; Sill, M; Jones, D T W; Hovestadt, V; Sturm, D; Koelsche, C; Bertoni, A; Schweizer, L; Korshunov, A; Weiß, E K; Schliesser, M G; Radbruch, A; Herold-Mende, C; Roth, P; Unterberg, A; Hartmann, C; Pietsch, T; Reifenberger, G; Lichter, P; Radlwimmer, B; Platten, M; Pfister, S 
M; von Deimling, A; Weller, M; Wick, W (2014). Integrated DNA methylation and copy-number profiling identify three clinically and biologically relevant groups of anaplastic glioma. Acta Neuropathologica, 128(4):561-571.

DOI: https://doi.org/10.1007/s00401-014-1315-x 
Integrated DNA methylation and copy-number profiling identifies three clinically and biologically relevant groups of anaplastic glioma

Benedikt Wiestler ${ }^{1,6 *}$, David Capper $^{2,7}$, Martin Sill ${ }^{11 *}$, David TW Jones ${ }^{9}$, Volker Hovestadt $^{10}$, Dominik Sturm ${ }^{9}$, Christian Koelsche ${ }^{2,7}$, Anna Bertoni ${ }^{10}$, Leonille Schweizer ${ }^{2,7}$, Andrey Korshunov ${ }^{2,7}$, Elisa K Weiß ${ }^{1,6}$, Maximilian G Schliesser ${ }^{6}$, Alexander Radbruch ${ }^{4}$, Christel Herold-Mende ${ }^{3}$, Patrick Roth ${ }^{16}$, Andreas Unterberg ${ }^{3}$, Christian Hartmann ${ }^{2,12}$, Torsten Pietsch $^{13}$, Guido Reifenberger ${ }^{14,15}$, Peter Lichter ${ }^{10}$, Bernhard Radlwimmer ${ }^{10}$, Michael Platten $^{1,8}$, Stefan M Pfister ${ }^{5,9}$, Andreas von Deimling ${ }^{2,7}$, Michael Weller $^{16}$, Wolfgang Wick ${ }^{1,6+}$ *These authors contributed equally to this manuscript

${ }^{1}$ Departments of Neurooncology, ${ }^{2}$ Neuropathology, ${ }^{3}$ Neurosurgery, ${ }^{4}$ Neuroradiology and ${ }^{5}$ Pediatric Oncology, Hematology, and Immunology, Heidelberg University Hospital and German Cancer Consortium (DKTK) and German Cancer Research Center (DKFZ), Clinical Cooperation Units ${ }^{6}$ Neurooncology, ${ }^{7}$ Neuropathology and ${ }^{8}$ Neuroimmunology and Brain Tumor Immunology, ${ }^{9}$ Division of Pediatric Neurooncology, ${ }^{10}$ Division of Molecular Genetics, ${ }^{11}$ Division of Biostatistics, 69120 Heidelberg; ${ }^{12}$ Department for Neuropathology, Institute of Pathology, Medical University of Hannover, 30625 Hannover; ${ }^{13}$ Department of Neuropathology, University of Bonn Medical Center, 53105 Bonn, all Germany; ${ }^{14}$ Department of Neuropathology, Heinrich-Heine-University and ${ }^{15}$ DKTK, partner site Essen/Düsseldorf, 40225 Düsseldorf, all Germany; ${ }^{16}$ Department of Neurology, University Hospital Zurich, Frauenklinikstrasse 26, CH-8091 Zurich, Switzerland

\section{${ }^{+}$Corresponding Author:}

Wolfgang Wick, Department of Neurooncology, Neurology Clinic \& National Center for Tumor Diseases, University of Heidelberg and German Cancer Research Center, Im 
Neuenheimer Feld 400, D-69120 Heidelberg, phone +49 (0)6221 56 7075, fax +49 (0)6221 56 7554, email: wolfgang.wick@ med.uni-heidelberg.de

Funding: The work was supported by the German Cancer Aid (Deutsche Krebshilfe, "Molecular classification of anaplastic gliomas in the NOA-04 trial", project 110624) to WW and MW and the Personalized Oncology Program of the NCT Heidelberg.

Key words: Anaplastic glioma, IDH, G-CIMP, 1p/19q, 450k 


\section{ABSTRACT}

The outcome of patients with anaplastic gliomas varies considerably. Whether a molecular classification of anaplastic gliomas based on large scale genomic or epigenomic analyses is superior to histopathology for reflecting distinct biological groups, predicting outcomes and guiding therapy decisions has yet to be determined.

Epigenome-wide DNA methylation analysis, using a platform which also allows the detection of copy-number aberrations, was performed in a cohort of 228 patients with anaplastic gliomas (astrocytomas, oligoastrocytomas and oligodendrogliomas), including 115 patients of the NOA-04 trial. We further compared these tumors with a group of 55 glioblastomas. Unsupervised clustering of DNA methylation patterns revealed two main groups correlated with $I D H$ status: $\mathrm{CpG}$ island methylator phenotype (CIMP) positive (77.5\%) or negative (22.5\%). CIMP ${ }^{\text {pos }}$ (IDH mutant) tumors showed a further separation based on copy-number status of chromosome arms $1 \mathrm{p}$ and $19 \mathrm{q} . \mathrm{CIMP}^{\text {neg }}$ (IDH wild type) tumors showed hallmark copy-number alterations of glioblastomas, and clustered together with CIMP ${ }^{\text {neg }}$ glioblastomas without forming separate groups based on WHO grade. Notably, there was no molecular evidence for a distinct biological entity representing anaplastic oligoastrocytoma. Tumor classification based on CIMP and 1p/19q status was significantly associated with survival, allowing a better prediction of outcome than the current histopathological classification: Patients with CIMP ${ }^{\text {pos }}$ tumors with $1 \mathrm{p} / 19 \mathrm{q}$ codeletion (CIMP-codel) had the best prognosis, followed by patients with CIMP ${ }^{\text {pos }}$ tumors but intact 1p/19q status (CIMP-non-codel). Patients with CIMP $^{\text {neg }}$ anaplastic gliomas (GBM-like) had the worst prognosis.

Collectively, our data suggest that anaplastic gliomas can be grouped by $I D H$ and $1 \mathrm{p} / 19 \mathrm{q}$ status into three molecular groups that show clear links to underlying biology and a significant association with clinical outcome in a prospective trial cohort. 


\section{INTRODUCTION}

Anaplastic gliomas of World Health Organization (WHO) grade III comprise a common primary CNS tumor in adults. They are histologically subdivided into anaplastic astrocytomas, oligodendrogliomas and mixed oligoastrocytomas according to morphological criteria [16]. While this histological classification has prognostic value [28], it is prone to high interobserver variation, especially for anaplastic oligoastrocytomas, but also for the differentiation between anaplastic glioma and glioblastoma (WHO grade IV) [13]. In recent years, large-scale genomic and epigenomic studies have greatly increased our insight into the biology of malignant gliomas, identifying key alterations and molecular groups $[4,8,23,26]$ which may complement the WHO classification. The discovery of a prognostically favorable point mutation in isocitrate dehydrogenase 1 (IDHI) codon 132 [19], resulting in a neomorphic enzymatic capacity to produce 2-hydroxyglutrate from $\alpha$-ketoglutarate, has considerably changed the understanding of glioma biology [27]. IDHI (and less frequently $I D H 2)$ mutations are rare $(\sim 5 \%)$ in primary glioblastomas, but are found in the majority of secondary glioblastomas as well as diffuse (WHO grade II) and anaplastic gliomas, especially in oligodendrogliomas [34]. Patients with $I D H$ mutant glioblastoma have a better prognosis than $I D H$ wild type anaplastic astrocytoma patients [9]. The $I D H$ mutation causes epigenetic remodeling [25], resulting in a $\mathrm{CpG}$ island methylator phenotype (CIMP) [18]. Indeed, gliomas across histological groups with an $I D H$ mutation carry similar epigenetic profiles [6]. This and other studies [14] suggest that $I D H$ mutant gliomas form a biologically distinct molecular group from their wild type counterparts. This notion is supported by the finding that methylation of the promoter of $O^{6}$-methylguanine DNA-methyltransferase (MGMT) is merely prognostic in $I D H$ mutant anaplastic gliomas, whereas it is predictive for response to alkylating chemotherapy in $I D H$ wild type gliomas [29, 31].

Besides $I D H$, it has been known for some time that patients with tumors harboring $1 \mathrm{p} / 19 \mathrm{q}$ codeletion have a better prognosis [22]. When two phase III trials reported their long-term 
follow-up, both demonstrating an overall survival benefit from combined treatment with radiotherapy and procarbazine, $\mathrm{CCNU}$ and vincristine $(\mathrm{PCV})$ chemotherapy mainly in patients with $1 \mathrm{p} / 19 \mathrm{q}$ codeleted tumors, the $1 \mathrm{p} / 19 \mathrm{q}$ codeletion gained predictive properties $[2,5]$. Mutations of the homolog of the Drosophila gene capicua $(C I C)$ on chromosome $19 \mathrm{q}$ and farupstream element binding protein $1(F U B P 1)$ on $1 \mathrm{p}$ have been identified as potential mechanisms involved in the biology of $1 \mathrm{p} / 19 \mathrm{q}$ codeleted gliomas [3]. Gene expression clustering plus $I D H 1$ and $1 \mathrm{p} / 19 \mathrm{q}$ status revealed molecular groups with prognostic value among oligodendroglial tumors [7].

Mutually exclusive mutations affecting telomerase reverse transcriptase (TERT) and alphathalassemia/mental retardation syndrome X-linked (ATRX) have been detected in malignant gliomas $[11,21]$. Two point mutations in the promoter of TERT (C228 and C250) resulting in higher TERT mRNA expression were discovered with high frequency in oligodendrogliomas (usually co-occurring with $1 \mathrm{p} / 19 \mathrm{q}$ codeletion) and primary glioblastomas. Alternative lengthening of telomeres (ALT) is another, telomerase-independent mechanism of telomere maintenance. ATRX mutations, usually leading to reduced or absent ATRX protein [15], have been linked to ALT [10]. Consequently, loss of ATRX expression predominantly occurs in astrocytomas and mixed oligoastrocytomas without 1p/19q codeletion (i.e. TERT wild type tumors) and seems to identify a prognostically more favorable molecular group among anaplastic astrocytoma patients [32].

Genome-wide sequencing data in 284 glioblastoma samples from the TCGA consortium showed that roughly $50 \%$ harbored at least one, usually mutually exclusive somatic mutation in genes functionally linked to chromatin organization, such as $I D H 1 / 2, S E T D 2, E Z H 2$, the HDAC family or ATRX [4]. Analysis of genome-wide DNA methylation patterns revealed distinct molecular groups of glioblastoma, associated with defined hotspot mutations (IDH and $H 3 F 3 A$, respectively) or common genomic aberrations like EGFR amplifications [24]. 
In contrast to glioblastomas, anaplastic gliomas have been analyzed less comprehensively [7, 17]. Here, the feasibility and prognostic value of a molecular classification of anaplastic gliomas based on epigenetic and copy-number analysis is investigated. 


\section{MATERIALS \& METHODS}

\section{Patients and tumor samples}

Primary tumor samples of anaplastic gliomas for DNA methylation analysis $(n=228)$ were collected at the Heidelberg University Hospital (Heidelberg, Germany, $\mathrm{n}=113$ ) and from the NOA-04 trial $(\mathrm{n}=115)[28]$.

\section{DNA methylation profiling}

For genome-wide assessment of DNA methylation patterns, tumor samples were subjected to microarray analyses at the Genomics and Proteomics Core Facility of the German Cancer Research Center (DKFZ) using the Infinium HumanMethylation450 BeadChip (Illumina, San Diego, CA).

The following filtering criteria were applied: Removal of probes targeting the $\mathrm{X}$ and $\mathrm{Y}$ chromosomes $(\mathrm{n}=11,551)$, removal of probes containing a single-nucleotide polymorphism (dbSNP132 Common) within five base pairs of and including the targeted $\mathrm{CpG}$ site $(\mathrm{n}=$ 24,536), and probes not mapping uniquely to the human reference genome (hg19) allowing for one mismatch $(\mathrm{n}=9,993)$. In total, 438,370 probes were kept for analysis.

\section{Statistical analysis of DNA methylation}

For unsupervised hierarchical clustering we selected the 10,000 most variable methylated probes that showed highest median absolute deviation (MAD) across beta values. Pairwise similarity between cases was calculated using euclidean distance and hierarchical clustering was performed using Ward's linkage method. To cluster CpG probes we applied hierarchical clustering using 1-centered Pearson correlation as similarity measure and average linkage as hierarchical clustering method.

To predict MGMT methylation using methylation (M-values) of CpG probes "cg12434587" and "cg12981137", we applied the logistic regression model MGMT-STP27 [1]. 


\section{Detection of TERT promoter and ATRX aberrations}

Data for TERT promoter hotspot mutations and for loss of ATRX expression (using immunohistochemistry (IHC)) in this patient cohort have been reported before [12, 32].

\section{Data repository}

Data are accessible at GEO (http://www.ncbi.nlm.nih.gov/geo/), accession number GSE58218. Additional methylation data of 55 adult glioblastoma samples used for comparison with anaplastic gliomas and six non-neoplastic brain tissue samples used as reference to generate copy-number variation plots are publicly available at GEO under the accession number GSE36278.

\section{Statistical analysis of clinical and molecular data}

Kaplan-Meier estimator, log rank test and multivariate Cox regression analyses were used to assess survival differences. Comparisons of binary and categorical patient characteristics between molecular groups were performed by the use of a two-sided Fisher's exact test. Continuous variables were compared using ANOVA and post-hoc pairwise comparisons with Holm's multiple testing procedure. Analyses were carried out using Stata IC version 12.1 (StataCorp LP, College Station, TX, USA) and R version 3.0.2 [20]. 


\section{RESULTS}

\section{Unsupervised analysis identifies two main clusters of anaplastic gliomas}

Unsupervised clustering methods using genome-wide DNA methylation patterns generated from 228 anaplastic gliomas revealed two main clusters, which were closely associated with IDH mutation status (Figure 1): In the first cluster, consisting of 177 (77.5\%) samples, 156/160 samples with available mutation status harbored an $I D H$ mutation (94\%, IDH status was unavailable for 17 samples). Only 1 out of 35 assessable samples (3\%) in the second cluster (in total 51 tumors, $22.5 \%$ ) carried an IDH mutation (IDH status was unavailable for 16 patients). In analogy to similar DNA methylation patterns observed in glioblastoma, we thus refer to the first (hypermethylated) cluster as CIMP ${ }^{\text {pos }}$ and to the second cluster as $\mathrm{CIMP}^{\text {neg }}$ (Supplementary Figure 1). Patients with CIMP ${ }^{\text {pos }}$ tumors had significantly longer overall survival than patients with a CIMP $^{\text {neg }}$ tumor $(\log$ rank $\mathrm{p}<0.001$; Supplementary Figure 2). Anaplastic astrocytomas were more frequently $\operatorname{CIMP}^{\text {neg }}(34 / 93,36.5 \%)$ than oligodendrogliomas $(10 / 71,14 \%)$ or oligoastrocytomas $(7 / 64,11 \%$; Fisher's exact test $\mathrm{p}<$ 0.001, Table 1).

The effect of $I D H$ mutation on the methylome is so pronounced that identification of further molecular groups beyond $\mathrm{CIMP}^{\text {pos }}$ and $\mathrm{CIMP}^{\text {neg }}$ is challenging when assessing all samples together. We therefore performed an additional analyses using copy-number and methylation data in order to investigate potential substructures within these two clusters, and also included previously published glioblastomas with known CIMP status [24].

\section{CIMP $^{\text {pos }}$ tumors are subtyped by 1 p/19q status}

Unsupervised hierarchical clustering of the 177 CIMP $^{\text {pos }}$ anaplastic gliomas and 14 CIMP $^{\text {pos }}$ / IDH mutant glioblastomas showed a clear separation of samples into two main clusters (Figure 2). Copy-number analysis revealed that this separation is based on $1 \mathrm{p} / 19 \mathrm{q}$ status, as $79 / 82$ samples $(96 \%)$ in one cluster had $1 \mathrm{p} / 19 \mathrm{q}$ codeletion as opposed to $8 / 109(7 \%)$ in the 
other cluster (Fisher's exact test, $\mathrm{p}<0.001$ ). As expected from the distribution of $1 \mathrm{p} / 19 \mathrm{q}$ codeletion in anaplastic gliomas, the group with $1 \mathrm{p} / 19 \mathrm{q}$ codeletion was significantly enriched for oligodendrogliomas and oligoastrocytomas, as was the group with intact $1 \mathrm{p} / 19 \mathrm{q}$ status for astrocytomas (Table 1). We therefore termed these molecular groups CIMP-codel and CIMPnon-codel, respectively. There was no further subtyping based on histology, e.g. a split between astrocytomas and oligoastrocytomas in the CIMP-non-codel cluster. None of the 14 $\mathrm{CIMP}^{\mathrm{pos}}$ glioblastomas displayed a $1 \mathrm{p} / 19 \mathrm{q}$ codeletion and all clustered with the CIMP-noncodel tumors without forming a separate molecular group. Furthermore, characteristic glioblastoma copy-number alterations (EGFR amplification, gain of chromosome 7 and loss of chromosome 10) were evenly rare in both $\mathrm{CIMP}^{\mathrm{pos}}$ molecular groups. Homozygous CDKN2A deletion occurred in 13/109 CIMP-non-codel tumors (two glioblastomas, 11 anaplastic gliomas) and in no CIMP-codel tumor. However, the low numbers precluded further statistical analysis of survival differences in the above mentioned smaller groups.

The biological differences between CIMP-non-codel and CIMP-codel tumors are further evidenced by the distribution of TERT promoter mutations and ATRX expression in the two molecular groups: TERT promoter mutations (C228 and C250) were detected in 57/65 CIMPcodel tumors (88\%), but only in 7/80 in the CIMP-non-codel group (9\%, notably, 6 of these had $1 \mathrm{p} / 19 \mathrm{q}$ codeletion). Conversely, loss of ATRX expression determined by immunohistochemistry was common in the CIMP-non-codel group, where 32/40 tumors (80\%) had lost ATRX expression as opposed to the CIMP-codel group, where only 1/34 tumors with available ATRX status showed this feature. Of note, no tumor (of 86 samples being analyzed both for TERT and ATRX status) displayed both, an ATRX loss and a TERT promoter mutation, consistent with these two pathways of telomere lengthening being mutually exclusive.

Interestingly, of the eight tumors with $1 \mathrm{p} / 19 \mathrm{q}$ codeletion that were found in the CIMP-noncodel group by cluster analysis, seven clustered tightly together and had TERT mutations. 
These tumors stably clustered in the CIMP-non-codel group even when using different similarity measures and linkage methods for the hierarchical clustering, indicating that these tumors, even while carrying $1 \mathrm{p} / 19 \mathrm{q}$ codeletion, have a different methylation profile than the majority of the $1 \mathrm{p} / 19 \mathrm{q}$ codeleted tumors. By histology, $6 / 7$ of these tumors were diagnosed as anaplastic oligodendroglioma.

Assessment of MGMT promoter methylation using the MGMT-STP27 algorithm [1] revealed a high frequency of $M G M T$ promoter methylation in CIMP ${ }^{\text {pos }}$ tumors: Of 191 tumors, only nine tumors $(5 \%)$ were predicted to have an unmethylated $M G M T$ promoter. These nine samples (eight anaplastic gliomas, one glioblastoma) all belonged to the CIMP-non-codel group, while all 82 tumors in the CIMP-codel group were scored as MGMT methylated. Importantly though, as the MGMT-STP27 was trained on glioblastoma DNA samples from fresh frozen tissue its ability to correctly predict the MGMT status of DNA samples coming from FFPE material might be limited (see Supplementary Discussion).

\section{CIMP $^{\text {neg }}$ anaplastic gliomas share copy-number aberrations and methylation profiles}

\section{with $I D H$ wild type glioblastomas}

As with the CIMP ${ }^{\text {pos }}$ tumors, we grouped the 51 CIMP $^{\text {neg }}$ anaplastic gliomas with 41 glioblastomas with known copy-number and methylation profiles from the study of Sturm et al. [24]. Unsupervised clustering did not separate anaplastic gliomas and glioblastomas into distinct clusters, with tumors of both WHO grades largely mixing (Figure 3). Subsets of CIMP $^{\text {neg }}$ anaplastic gliomas clustered together with glioblastomas of each of the mesenchymal, RTK I and RTK II groups, respectively. The seeming interposition of the RTK I samples in between RTK II samples is most likely caused by different normal cell amounts and/or hybridization quality and not a real biological difference between the two RTK II clusters. This biological similarity of CIMP $^{\text {neg }}$ anaplastic gliomas and glioblastomas based on methylation data is also reflected in the copy-number profiles: hallmark glioblastoma 
alterations were found at similar frequency in anaplastic gliomas and glioblastomas (Fisher's exact test, $\mathrm{p}>0.1$ for each comparison; Figure 3). Furthermore, copy-number aberrations showed typical patterns, for example with EGFR amplifications and $C D K N 2 A$ deletions occurring mostly in RTK II glioblastomas as well as the anaplastic gliomas clustering with them. MGMT promoter methylation was also found at similar frequency in both WHO grades: 23/51 anaplastic gliomas (45\%) and 18/41 glioblastomas (44\%) were predicted to have a methylated $M G M T$ promoter according to the $M G M T$-STP27 algorithm. In CIMP ${ }^{\text {neg }}$ samples from NOA-04 $(\mathrm{n}=24), M G M T$ status clearly was associated with differences in progressionfree survival in the chemotherapy arm, while no such obvious effect was seen in the radiotherapy arm (Supplementary Figure 3). However, the small number of samples in each subgroup precluded further statistical analysis.

\section{Integrated molecular classification is significantly associated with survival}

Taken together, these data suggest that anaplastic gliomas can be robustly divided into three main molecular groups based on DNA methylation and copy-number data and independent of histology: CIMP $^{\text {neg }}$ tumors, which molecularly resemble glioblastomas, CIMP-non-codel tumors with intact 1p/19q and CIMP-codel tumors with 1p/19q codeletion (Figure 5).

Our series of anaplastic gliomas contained 115 samples from the biomarker cohort of the NOA-04 trial, which investigated the optimal sequence of radio- and chemotherapy in newlydiagnosed anaplastic gliomas [28]. Baseline patient characteristics of these 115 samples closely resembled those of the entire NOA-04 trial cohort (Supplementary Table 1), except for an enrichment of patients with tumor resection as opposed to biopsy due to the tissue requirements for DNA methylation analyses. As observed in the entire NOA-04 cohort, both treatment sequences performed very similar (log rank test, $\mathrm{p}=0.94)$.

Of the 115 samples, 24 were CIMP ${ }^{\text {neg }}, 48$ CIMP-non-codel and 43 CIMP-codel. Time to treatment failure (TTF), the primary endpoint of NOA-04, showed a clear separation of 
patients based on molecular classification (Figure 4a): CIMP-codel tumors had the best prognosis (median TTF not reached [95\% CI 1840 days - not reached]), followed by CIMPnon-codel tumors (median TTF 1691 days [95\% CI 793 - 2315 days]), while CIMP ${ }^{\text {neg }}$ tumors had the shortest median TTF (496 days [95\% CI 326 - 1071 days]). This was also true for overall survival (Figure 4b). As opposed to reference histology (Figure 4c,d), this led to a clearer separation of prognostic subgroups. Consequently, in a multivariate Cox regression model including molecular classification, reference histology and age, only molecular classification and age, but not reference histology, were significantly associated with outcome (Table 2). To account for potential collinearity between molecular classification and reference histology, we analyzed the prediction error curves for Cox models based on reference histology or molecular classification only or including both. This indicated again a better prediction of survival through the molecular classification as opposed to reference histology and only a small improvement of prediction when including both reference histology and molecular classification (Supplementary Figure 4). 


\section{DISCUSSION}

Using DNA methylation profiling and copy-number analysis, we identified three principal molecular groups of anaplastic gliomas in a cohort of 228 samples: CIMP ${ }^{\text {neg }}$ tumors, which resemble glioblastomas, CIMP-codel tumors with $1 \mathrm{p} / 19 \mathrm{q}$ codeletion as a hallmark genomic alteration and CIMP-non-codel tumors, which lack this copy-number aberration (key characteristics are summarized in Table 1); the classification scheme is shown in Figure 5. Importantly, this classification not only reflects molecular differences between these groups, but also better translates into clinically relevant survival differences when compared with the WHO classification as evidenced in the biomarker cohort of the NOA-04 trial (Figure 4, Table 2).

The majority of oligoastrocytomas and oligodendrogliomas [34] belonged to the CIMP $\mathrm{P}^{\text {pos }}$ cluster. Based on their epigenome-wide DNA methylation profile, tumors belonging to the CIMP $^{\text {pos }}$ cluster can be further subdivided by $1 p / 19 q$ status (also the first split to occur after CIMP positive vs. negative when looking at all samples; Figure 1) as suggested before for a smaller $(n=46)$ series of purely oligodendroglial tumors [17]. We termed these clusters CIMP-codel (mainly 1p/19q codeleted with oligodendroglial histology) and CIMP-non-codel (mainly $1 \mathrm{p} / 19 \mathrm{q}$ intact and astrocytic). Notably, the $14 \mathrm{CIMP}^{\mathrm{pos}}$ glioblastomas included in this analysis all clustered well within the CIMP-non-codel molecular group. On the other hand, $\mathrm{CIMP}^{\text {neg }}$ (and hence IDH wild type) anaplastic gliomas were molecularly indistinguishable from CIMP ${ }^{\text {neg }}$ glioblastomas: Hallmark glioblastoma copy-number aberrations were found in comparable frequency in both tumor grades. Consequently, unsupervised clustering did not sort WHO grade III and IV CIMP ${ }^{\text {neg }}$ tumors into separate clusters. In contrast, these copynumber aberrations are rare in CIMP ${ }^{\text {pos }}$ tumors (of WHO grade III or IV). Thus, anaplastic gliomas and glioblastomas with a given CIMP status are more similar to each other than $\mathrm{CIMP}^{\text {pos }}$ vs. $\mathrm{CIMP}^{\text {neg }}$ tumors of a certain histology, strongly supporting the notion that IDH mutant and $I D H$ wild type tumors are in fact distinct entities [14]. 
In our cohort, median overall survival was longer in $\mathrm{CIMP}^{\text {neg }}$ anaplastic gliomas than in glioblastomas (Supplementary Figure 5). However, the cohort for this comparison is very heterogeneous with respect to treatment and clinical follow-up, including prognostically favorable NOA-04 study patients. Longer overall survival for IDH wild type anaplastic astrocytomas compared to glioblastomas has been reported in another retrospective series [9]. However, in elderly patients with newly diagnosed WHO grade III or IV astrocytomas, which are almost always $I D H$ wild type [33], there was no survival difference [30]. Since most anaplastic gliomas eventually progress to secondary glioblastomas at recurrence, a survival difference may just reflect that CIMP ${ }^{\text {neg }}$ anaplastic gliomas are diagnosed in an earlier stage of tumor development compared to CIMP ${ }^{\text {neg }}$ glioblastoma. Another explanation may be yet to be defined groups in the CIMP ${ }^{\text {neg }}$ anaplastic gliomas which (i) share a glioblastoma-like clinical course or show (ii) a markedly longer survival. For the time being, our findings suggest that WHO grading retains a prognostic role within the group of CIMP ${ }^{\text {neg }}$ high-grade gliomas.

Histological classification of anaplastic gliomas, in particular of mixed oligoastrocytomas, has considerable interobserver variability [13]. Molecularly, oligoastrocytomas do not form a separate biological entity, as they are evenly distributed between the CIMP-codel and CIMPnon-codel clusters (24 and 33 cases, respectively), while pure oligodendrogliomas and astrocytomas were predominantly sorted in the CIMP-codel or CIMP-non-codel cluster, respectively (Table 1). Since there is no further subtyping based on histology in either CIMP $^{\text {pos }}$ cluster, e.g. a split between astrocytomas and oligoastrocytomas in the CIMP-noncodel cluster, there seems to be no clear biological basis for the diagnosis of an oligoastrocytoma. Mean age at diagnosis significantly differed between all three groups (Table 1, $\mathrm{p}<0.0001$, ANOVA).

With a stringent central histology, NOA-04 demonstrated a clear survival benefit for patients with oligodendrogliomas and oligoastrocytomas compared to astrocytomas, which was also retained in our cohort of 115 NOA-04 patients (Supplementary Table 2). This cohort is 
therefore well suited to compare the clinical significance of this molecular classification of anaplastic gliomas. In a multivariate Cox regression model, histological assessment did not significantly correlate with survival anymore once molecular subtyping was added as a covariate, pointing to a diagnostic superiority of molecular subtyping.

In the long-term follow-up analysis of two large randomized phase III trials, $1 \mathrm{p} / 19 \mathrm{q}$ codeletion was concordantly shown to predict benefit from radio-chemotherapy $[2,5]$. However, in both trials several patients with $1 \mathrm{p} / 19 \mathrm{q}$ codeletion did not benefit from this intensified treatment. In our classification, while $1 \mathrm{p} / 19$ status is clearly the defining feature for the two molecular groups, 8 out of 95 tumors in the CIMP-non-codel group carried 1p/19q codeletion (and TERT mutation). Whether these tumors represent a distinct group is currently unclear. An influence of other factors on the DNA methylation profile, such as infiltrating normal (reactive) astrocytes can also not be excluded. Based on this observation, however, it seems possible that these might be the rare $1 \mathrm{p} / 19 \mathrm{q}$ codeleted tumors not benefitting from intensified treatment. This hypothesis could be tested in either RTOG-9402 or EORTC26951. Furthermore, both trials were designed to include only oligodendroglial tumors, but no astrocytomas, although reference histology revealed a relevant proportion of grade II gliomas, anaplastic astrocytoma and even glioblastoma [13]. While $1 \mathrm{p} / 19 \mathrm{q}$ codeletion occurs only infrequently in astrocytomas (three out of 93 anaplastic astrocytomas belonged to the CIMPcodel group), our observation that anaplastic astrocytoma with $1 \mathrm{p} / 19 \mathrm{q}$ codeletion molecularly resemble codeleted oligodendroglial tumors supports the notion that this subset should also be treated with combined radio-chemotherapy at initial diagnosis.

In summary, we demonstrate a classification of anaplastic gliomas based on (epi)genomewide DNA methylation and copy-number profiles, where tumors can be divided into CIMP ${ }^{\text {pos }}$ tumors with or without $1 \mathrm{p} / 19 \mathrm{q}$ loss, and $\mathrm{CIMP}^{\text {neg }}$ tumors which closely resemble glioblastomas (and may in fact be true glioblastomas biologically, but diagnosed at an earlier stage of tumor development, for example). This information, together with $M G M T$ status, can 
be readily obtained from one platform (450k DNA methylation array), suggesting a possible simplification of routine diagnostic testing for this set of tumors. This biological classification is also significantly associated with outcome: patients with CIMP-codel tumors have the best prognosis, followed by those with CIMP-non-codel tumors and lastly CIMP ${ }^{\text {neg }}$ tumors. Defining such groups will allow for a clearer understanding of their pathophysiological drivers, enabling a more rational search for therapeutic targets. The presented molecular classification has the potential to greatly complement the standard histological WHO classification by providing a biologically meaningful and clinically relevant stratification algorithm, and warrants further examination in future clinical trials. 


\section{REFERENCES}

1. Bady P, Sciuscio D, Diserens A-C, Bloch J, van den Bent MJ, et al. (2012) MGMT methylation analysis of glioblastoma on the Infinium methylation BeadChip identifies two distinct $\mathrm{CpG}$ regions associated with gene silencing and outcome, yielding a prediction model for comparisons across datasets, tumor grades, and CIMP-status. Acta Neuropathol. 124:547-560

2. Van den Bent MJ, Brandes AA, Taphoorn MJB, Kros JM, Kouwenhoven MCM, et al. (2013) Adjuvant procarbazine, lomustine, and vincristine chemotherapy in newly diagnosed anaplastic oligodendroglioma: long-term follow-up of EORTC brain tumor group study 26951. J. Clin. Oncol. 31:344-350

3. Bettegowda C, Agrawal N, Jiao Y, Sausen M, Wood LD, et al. (2011) Mutations in CIC and FUBP1 contribute to human oligodendroglioma. Science 333:1453-1455

4. Brennan CW, Verhaak RGW, McKenna A, Campos B, Noushmehr H, et al. (2013) The somatic genomic landscape of glioblastoma. Cell 155:462-477

5. Cairncross G, Wang M, Shaw E, Jenkins R, Brachman D, et al. (2013) Phase III trial of chemoradiotherapy for anaplastic oligodendroglioma: long-term results of RTOG 9402. J. Clin. Oncol. 31:337-343

6. Christensen BC, Smith AA, Zheng S, Koestler DC, Houseman EA, et al. (2011) DNA methylation, isocitrate dehydrogenase mutation, and survival in glioma. J. Natl. Cancer Inst. 103:143-153

7. Erdem-Eraslan L, Gravendeel LA, de Rooi J, Eilers PHC, Idbaih A, et al. (2013) Intrinsic molecular subtypes of glioma are prognostic and predict benefit from adjuvant procarbazine, lomustine, and vincristine chemotherapy in combination with other prognostic factors in anaplastic oligodendroglial brain tumors: a report from EORTC stu. J. Clin. Oncol. 31:328-336

8. Frattini V, Trifonov V, Chan JM, Castano A, Lia M, et al. (2013) The integrated landscape of driver genomic alterations in glioblastoma. Nat. Genet. 45:1141-1149

9. Hartmann C, Hentschel B, Wick W, Capper D, Felsberg J, et al. (2010) Patients with IDH1 wild type anaplastic astrocytomas exhibit worse prognosis than IDH1-mutated glioblastomas, and IDH1 mutation status accounts for the unfavorable prognostic effect of higher age: implications for classification of gliomas. Acta Neuropathol. 120:707718

10. Heaphy CM, de Wilde RF, Jiao Y, Klein AP, Edil BH, et al. (2011) Altered telomeres in tumors with ATRX and DAXX mutations. Science 333:425

11. Killela PJ, Reitman ZJ, Jiao Y, Bettegowda C, Agrawal N, et al. (2013) TERT promoter mutations occur frequently in gliomas and a subset of tumors derived from cells with low rates of self-renewal. Proc. Natl. Acad. Sci. U. S. A. 110:6021-6026 
12. Koelsche C, Sahm F, Capper D, Reuss D, Sturm D, et al. (2013) Distribution of TERT promoter mutations in pediatric and adult tumors of the nervous system. Acta Neuropathol. 126:907-915

13. Kros JM, Gorlia T, Kouwenhoven MC, Zheng P-P, Collins VP, et al. (2007) Panel review of anaplastic oligodendroglioma from European Organization For Research and Treatment of Cancer Trial 26951: assessment of consensus in diagnosis, influence of 1p/19q loss, and correlations with outcome. J. Neuropathol. Exp. Neurol. 66:545-551

14. Lai A, Kharbanda S, Pope WB, Tran A, Solis OE, et al. (2011) Evidence for sequenced molecular evolution of IDH1 mutant glioblastoma from a distinct cell of origin. J. Clin. Oncol. 29:4482-4490

15. Liu X-Y, Gerges N, Korshunov A, Sabha N, Khuong-Quang D-A, et al. (2012) Frequent ATRX mutations and loss of expression in adult diffuse astrocytic tumors carrying IDH1/IDH2 and TP53 mutations. Acta Neuropathol. 124:615-625

16. Louis DN, Ohgaki H, Wiestler OD, Cavenee WK, Burger PC, et al. (2007) The 2007 WHO classification of tumours of the central nervous system. Acta Neuropathol. 114:97-109

17. Mur P, Mollejo M, Ruano Y, de Lope ÁR, Fiaño C, et al. (2013) Codeletion of 1p and $19 \mathrm{q}$ determines distinct gene methylation and expression profiles in IDH-mutated oligodendroglial tumors. Acta Neuropathol. 126:277-289

18. Noushmehr H, Weisenberger DJ, Diefes K, Phillips HS, Pujara K, et al. (2010) Identification of a $\mathrm{CpG}$ island methylator phenotype that defines a distinct subgroup of glioma. Cancer Cell 17:510-522

19. Parsons DW, Jones S, Zhang X, Lin JC-H, Leary RJ, et al. (2008) An integrated genomic analysis of human glioblastoma multiforme. Science 321:1807-1812

20. R Core Team (2013) R: A language and environment for statistical computing. R Foundation for Statistical Computing, Vienna, Austria. http://www.R-project.org/.

21. Schwartzentruber J, Korshunov A, Liu X-Y, Jones DTW, Pfaff E, et al. (2012) Driver mutations in histone $\mathrm{H} 3.3$ and chromatin remodelling genes in paediatric glioblastoma. Nature 482:226-231

22. Smith JS, Perry A, Borell TJ, Lee HK, O'Fallon J, et al. (2000) Alterations of chromosome arms $1 p$ and $19 q$ as predictors of survival in oligodendrogliomas, astrocytomas, and mixed oligoastrocytomas. J. Clin. Oncol. 18:636-645

23. Sturm D, Bender S, Jones DTW, Lichter P, Grill J, et al. (2014) Paediatric and adult glioblastoma: multiform (epi)genomic culprits emerge. Nat. Rev. Cancer 14:92-107

24. Sturm D, Witt H, Hovestadt V, Khuong-Quang D-A, Jones DTW, et al. (2012) Hotspot mutations in H3F3A and IDH1 define distinct epigenetic and biological subgroups of glioblastoma. Cancer Cell 22:425-437 
25. Turcan S, Rohle D, Goenka A, Walsh LA, Fang F, et al. (2012) IDH1 mutation is sufficient to establish the glioma hypermethylator phenotype. Nature 483:479-483

26. Verhaak RGW, Hoadley KA, Purdom E, Wang V, Qi Y, et al. (2010) Integrated genomic analysis identifies clinically relevant subtypes of glioblastoma characterized by abnormalities in PDGFRA, IDH1, EGFR, and NF1. Cancer Cell 17:98-110

27. Ward PS, Patel J, Wise DR, Abdel-Wahab O, Bennett BD, et al. (2010) The common feature of leukemia-associated IDH1 and IDH2 mutations is a neomorphic enzyme activity converting alpha-ketoglutarate to 2-hydroxyglutarate. Cancer Cell 17:225-234

28. Wick W, Hartmann C, Engel C, Stoffels M, Felsberg J, et al. (2009) NOA-04 randomized phase III trial of sequential radiochemotherapy of anaplastic glioma with procarbazine, lomustine, and vincristine or temozolomide. J. Clin. Oncol. 27:58745880

29. Wick W, Meisner C, Hentschel B, Platten M, Schilling A, et al. (2013) Prognostic or predictive value of MGMT promoter methylation in gliomas depends on IDH1 mutation. Neurology 81:1515-1522

30. Wick W, Platten M, Meisner C, Felsberg J, Tabatabai G, et al. (2012) Temozolomide chemotherapy alone versus radiotherapy alone for malignant astrocytoma in the elderly: the NOA-08 randomised, phase 3 trial. Lancet Oncol. 13:707-715

31. Wick W, Weller M, van den Bent M, Sanson M, Weiler M, et al. (2014) MGMT testing in neurooncology - A paradigm for prospects and challenges of biomarkerbased treatment decisions. Nat. Rev. Neurol. In press

32. Wiestler B, Capper D, Holland-Letz T, Korshunov A, von Deimling A, et al. (2013) ATRX loss refines the classification of anaplastic gliomas and identifies a subgroup of IDH mutant astrocytic tumors with better prognosis. Acta Neuropathol. 126:443-451

33. Wiestler B, Claus R, Hartlieb SA, Schliesser MG, Weiss EK, et al. (2013) Malignant astrocytomas of elderly patients lack favorable molecular markers: an analysis of the NOA-08 study collective. Neuro. Oncol. 15:1017-1026

34. Yan H, Parsons DW, Jin G, McLendon R, Rasheed BA, et al. (2009) IDH1 and IDH2 mutations in gliomas. N. Engl. J. Med. 360:765-773 


\section{FIGURE LEGENDS}

Figure 1. DNA methylation profiling separates anaplastic gliomas into two main clusters based on IDH status. Heatmap of DNA methylation levels in 228 anaplastic gliomas. Each row represents a probe and each column a sample. The level of DNA methylation (beta-value) is represented with a color scale as depicted. For each sample, $I D H$ mutation and $1 \mathrm{p} / 19 \mathrm{q}$ copy-number status as well as histology are indicated.

Figure 2. CIMP ${ }^{\text {pos }}$ anaplastic gliomas form two separate molecular subypes closely associated with $1 \mathbf{p} / 19 q$ status. DNA methylation heatmap showing the result of an unsupervised clustering of $177 \mathrm{CIMP}^{\text {pos }}$ anaplastic gliomas and $14 \mathrm{CIMP}^{\text {pos }}$ glioblastomas. Below, IDH, 1p/19q, TERT, ATRX and MGMT promoter methylation status and histology are given.

Figure 3. Unsupervised clustering analysis of DNA methylation and copy-number profiles of CIMP $^{\text {neg }}$ anaplastic gliomas $(n=51)$ and CIMP $^{\text {neg }}$ glioblastomas $(n=41)$ reveals a close biological similarity. For each sample, $I D H$ mutation status, copy-number aberrations, MGMT promoter methylation status and WHO grade are given. Additionally, cluster membership within the six glioblastoma clusters defined by Sturm et al. are given.

Figure 4. Molecular classification of anaplastic gliomas is a better prognosticator than the WHO classification. Kaplan-Meier plots for time to treatment failure (a, c) and overall survival $(b, d)$ for the 115 NOA-04 trial samples according to molecular classification $(a, b)$ or reference histology (c, d) are shown.

Figure 5. Summary of the proposed molecular classification. CNVs, copy-number variations. 

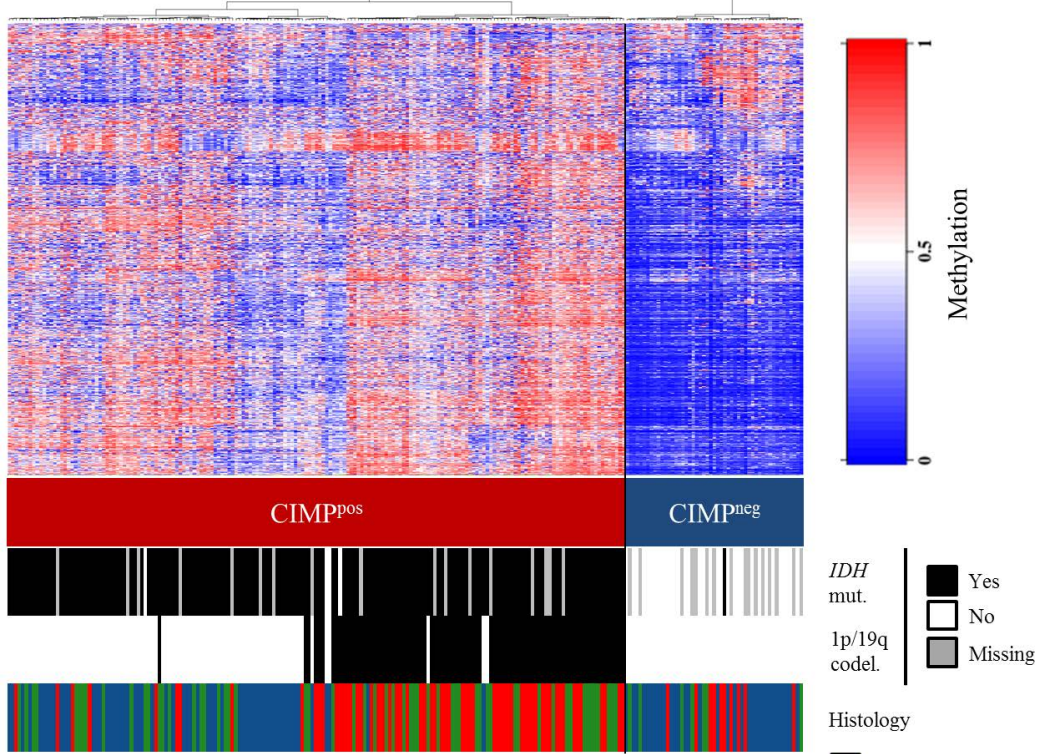

Histology

Astrocytoma

Oligoastrocytoma

Oligodendroglioma 


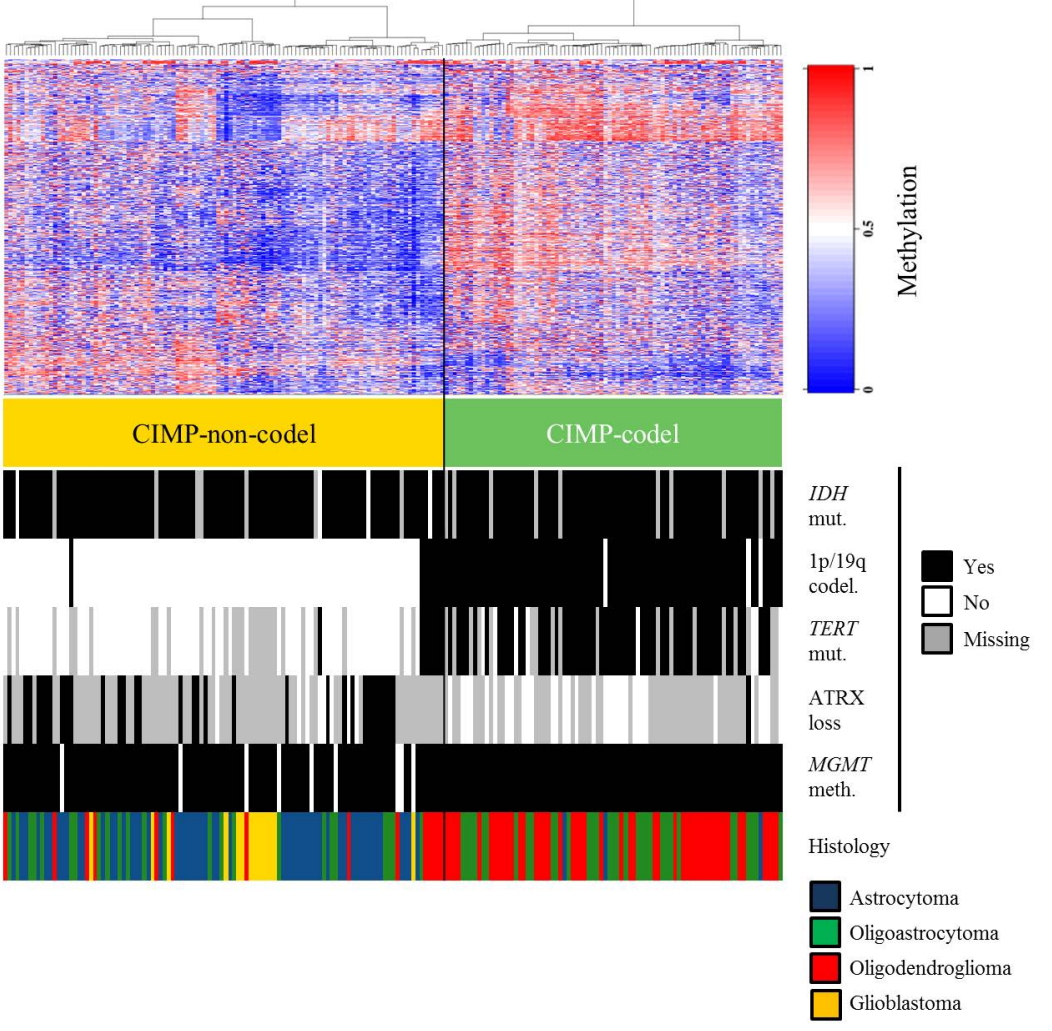




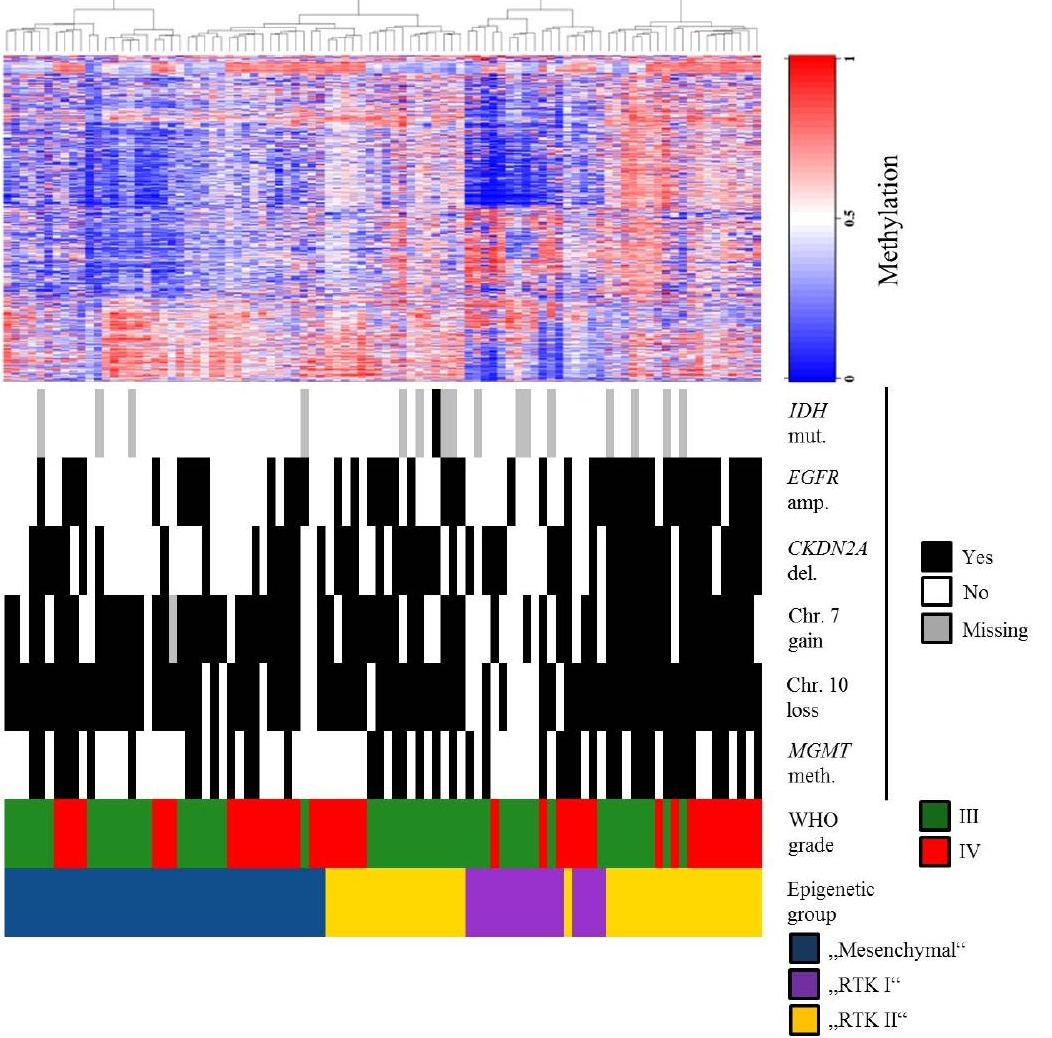




\section{Molecular classification}

a)

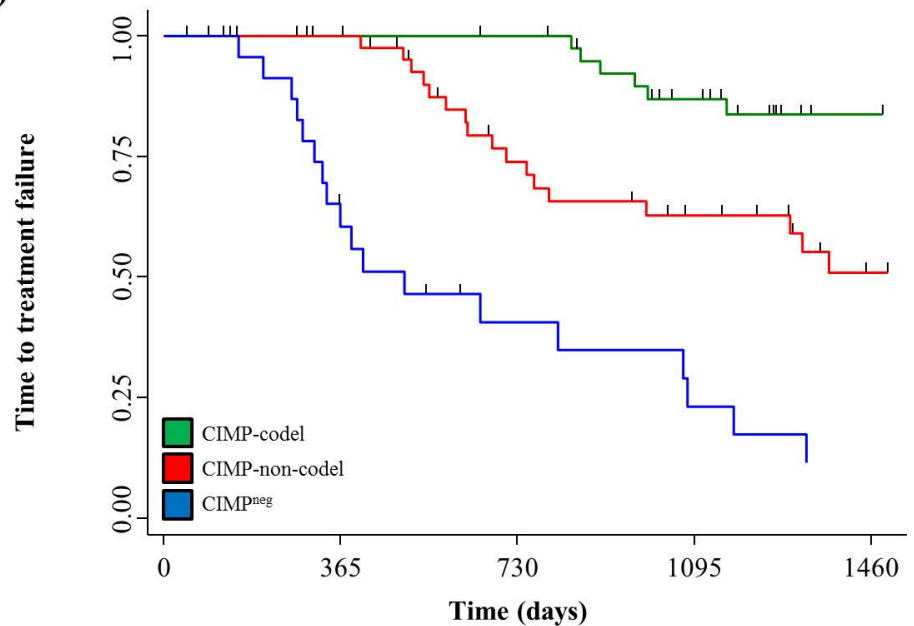

Number at risk

$\begin{array}{ll}\text { CIMP-O } & 43 \\ \text { CIMP-A } & 48\end{array}$

CIMPneg 24

41
38

38
10

33

22
6

b)

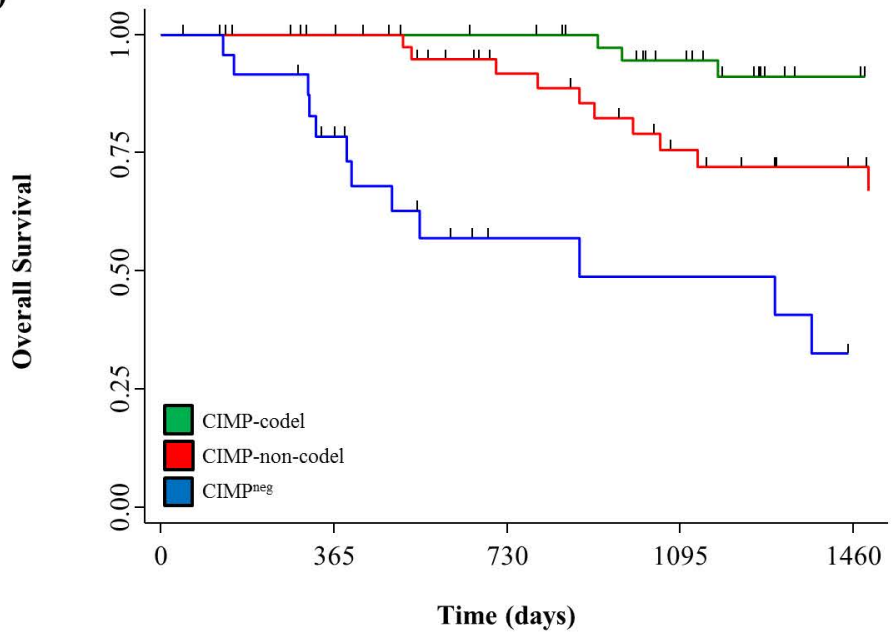

c)

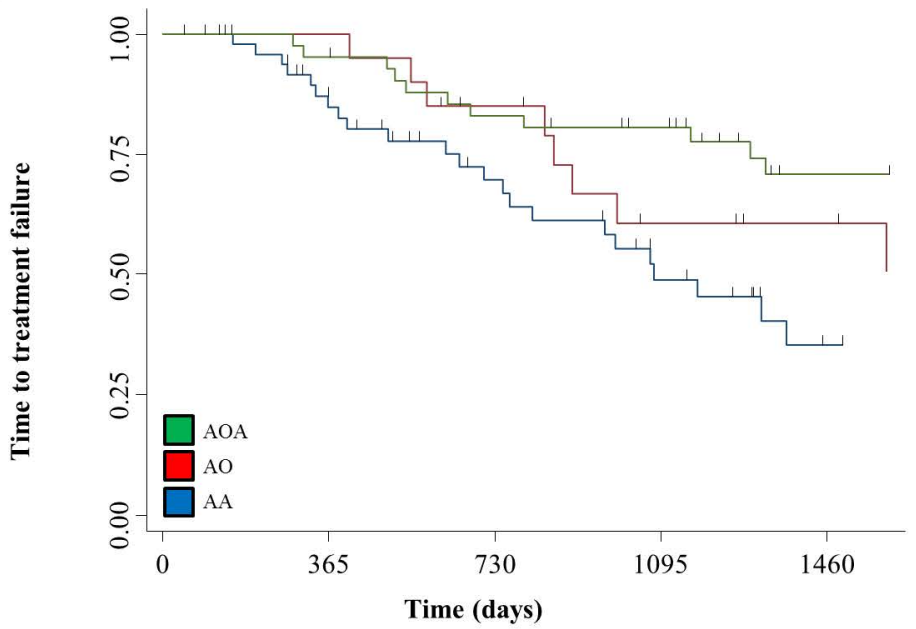

Number at risk

$\begin{array}{rr}\text { AA } & 50 \\ \text { AO } & 20 \\ \text { AOA } & 45\end{array}$

32

$\mathrm{AOA} 45$

d)

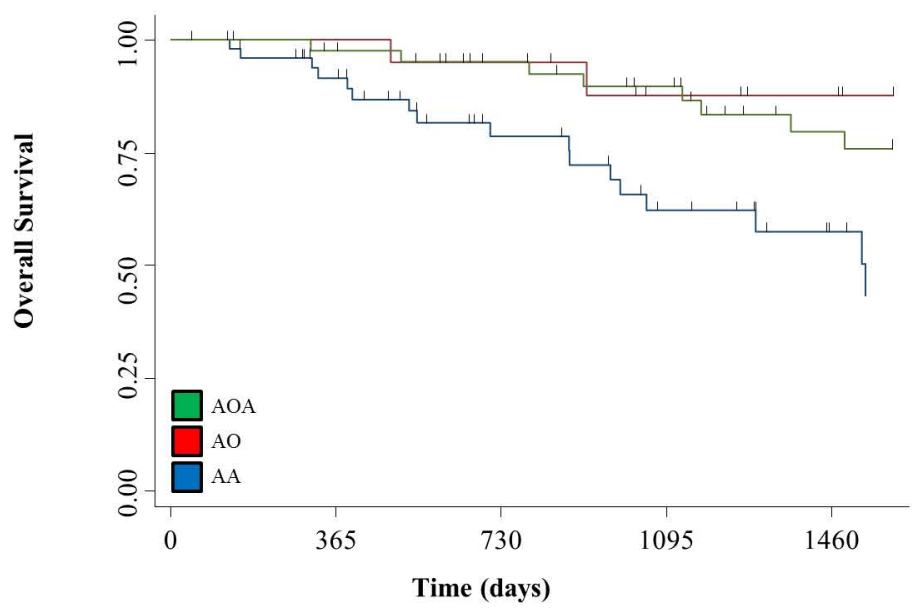

Number at risk

$\begin{array}{ll}\text { CIMP-O } & 43 \\ \text { CIMP-A } & 48\end{array}$

CIMPneg 24

$\begin{array}{cc}35 & 18 \\ 24 & 13 \\ 6 & 3\end{array}$

\section{Number at risk}

$$
\begin{array}{rr}
\text { AA } & 50 \\
\text { AO } & 20 \\
\text { AOA } & 45
\end{array}
$$




\section{Anaplastic glioma}

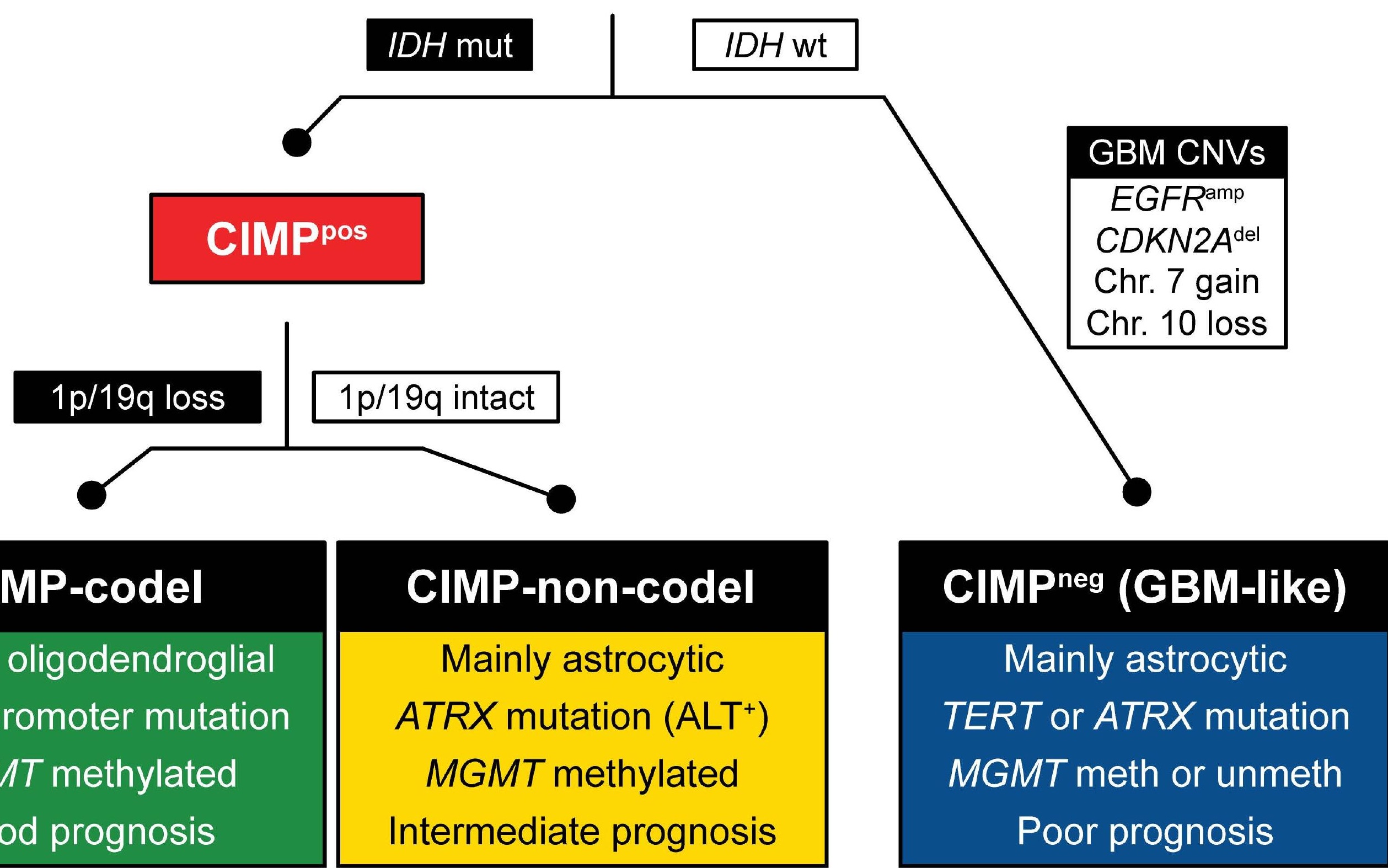

\section{Mainly oligodendroglial}

TERT promoter mutation

MGMT methylated

Good prognosis

\section{CIMP-non-codel}

Mainly astrocytic ATRX mutation $\left(\mathrm{ALT}^{+}\right)$ MGMT methylated Intermediate prognosis

\section{CIMPreg (GBM-like)}

Mainly astrocytic TERT or ATRX mutation MGMT meth or unmeth Poor prognosis 
TABLES

Table 1: Key characteristics of molecular groups

\begin{tabular}{|c|c|c|c|}
\hline & $\begin{array}{l}\text { CIMP-non-codel } \\
\text { (1p/19q intact) }\end{array}$ & $\begin{array}{c}\text { CIMP-codel } \\
\text { (1p/19q codeleted) }\end{array}$ & $\begin{array}{c}\text { CIMPneg } \\
(I D H \text { wild type })\end{array}$ \\
\hline \multicolumn{4}{|l|}{ Histology } \\
\hline Astrocytoma & $56(51 \%)$ & $3(4 \%)$ & $34(37 \%)$ \\
\hline Oligodendroglioma & $15(14 \%)$ & $46(56 \%)$ & $10(11 \%)$ \\
\hline Oligoastrocytoma & $24(22 \%)$ & $33(40 \%)$ & $7(7 \%)$ \\
\hline Glioblastoma & $14(13 \%)$ & 0 & $41(45 \%)$ \\
\hline $\begin{array}{l}\text { Mean age (years), } \\
(95 \% \mathrm{CI})\end{array}$ & $\begin{array}{c}38.7 \\
(36.6-40.7)\end{array}$ & $\begin{array}{c}45.7 \\
(43.4-48.1)\end{array}$ & $\begin{array}{c}49.3 \\
(46.9-51.8)\end{array}$ \\
\hline \multicolumn{4}{|l|}{ IDH } \\
\hline Mutated & $97(96 \%)$ & $73(100 \%)$ & $1(1.5 \%)$ \\
\hline Wild type & $4(4 \%)$ & 0 & $75(98.5 \%)$ \\
\hline Not available & 8 & 9 & 16 \\
\hline \multicolumn{4}{|l|}{$1 p / 19 q$} \\
\hline Codeleted & $8(7 \%)$ & $79(96 \%)$ & 0 \\
\hline Intact & $101(93 \%)$ & $3(4 \%)$ & $92(100 \%)$ \\
\hline \multicolumn{4}{|l|}{$T E R T$ promoter } \\
\hline Mutated & $7(9 \%)$ & $57(88 \%)$ & $21(49 \%)$ \\
\hline Wild type & $73(91 \%)$ & $8(12 \%)$ & $22(51 \%)$ \\
\hline Not available & 29 & 17 & 49 \\
\hline \multicolumn{4}{|l|}{ ATRX } \\
\hline Lost & $32(80 \%)$ & $1(3 \%)$ & $2(10 \%)$ \\
\hline Expressed & $8(20 \%)$ & $33(97 \%)$ & $20(90 \%)$ \\
\hline
\end{tabular}


ANEU-D-14-00273R -2-

\begin{tabular}{|c|c|c|c|}
\hline Not available & 69 & 48 & 70 \\
\hline \multicolumn{4}{|l|}{$M G M T$} \\
\hline Methylated & $100(92 \%)$ & $82(100 \%)$ & $41(45 \%)$ \\
\hline Unmethylated & $9(8 \%)$ & 0 & $51(55 \%)$ \\
\hline \multicolumn{4}{|c|}{ EGFR amplification } \\
\hline Yes & $2(2 \%)$ & $1(1.5 \%)$ & $45(59 \%)$ \\
\hline No & $107(98 \%)$ & $81(98.5 \%)$ & $47(51 \%)$ \\
\hline \multicolumn{4}{|c|}{$C D K N 2 A$ deletion } \\
\hline Yes & $13(12 \%)$ & 0 & $51(55.5 \%)$ \\
\hline No & $96(88 \%)$ & $82(100 \%)$ & $41(44.5 \%)$ \\
\hline \multicolumn{4}{|l|}{ Chr. 7 gain } \\
\hline Yes & $3(2.5 \%)$ & $2(2.5 \%)$ & $67(73.5 \%)$ \\
\hline No & $106(97.5 \%)$ & $80(97.5 \%)$ & $24(26.5 \%)$ \\
\hline \multicolumn{4}{|l|}{ Chr. 10 loss } \\
\hline Yes & $1(1 \%)$ & 0 & $77(83 \%)$ \\
\hline No & $108(99 \%)$ & $82(100 \%)$ & $15(17 \%)$ \\
\hline
\end{tabular}


Table 2: Multivariate Cox PH regression analysis for TTF in the NOA-04 cohort

\begin{tabular}{|c|c|c|c|}
\hline Variable & HR & 95\% CI & p \\
\hline AO vs. AA & 1.76 & $0.72-4.12$ & 0.211 \\
\hline AA vs. AOA & 1.85 & $0.9-3.84$ & 0.093 \\
\hline CIMP-non-codel $v s$. & 5.27 & $2.0-13.9$ & $\mathbf{0 . 0 0 1}$ \\
CIMP-codel & & & \\
\hline CIMP & & & \\
\hline CIMP-codel & & $5.02-31.32$ & $\mathbf{0 . 0 0 1}$ \\
\hline Age, per year increase & 12.54 & & $\mathbf{0 . 0 0 1}$ \\
\hline
\end{tabular}

AA, anaplastic astrocytoma; AO, anaplastic oligodendrogliomas; AOA, anaplastic oligoastrocytoma 


\section{SUPPLEMENTARY INFORMATION}

Integrated DNA methylation and copy-number profiling identifies three clinically and biologically relevant groups of anaplastic glioma

Benedikt Wiestler ${ }^{1,6 *}$, David Capper $^{2,7}$, Martin Sill ${ }^{11 *}$, David TW Jones ${ }^{9}$, Volker Hovestadt $^{10}$, Dominik Sturm ${ }^{9}$, Christian Koelsche ${ }^{2,7}$, Anna Bertoni ${ }^{10}$, Leonille Schweizer ${ }^{2,7}$, Andrey Korshunov ${ }^{2,7}$, Elisa K Weiß ${ }^{1,6}$, Maximilian G Schliesser ${ }^{6}$, Alexander Radbruch ${ }^{4}$, Christel Herold-Mende ${ }^{3}$, Patrick Roth ${ }^{16}$, Andreas Unterberg ${ }^{3}$, Christian Hartmann ${ }^{2,12}$, Torsten Pietsch $^{13}$, Guido Reifenberger ${ }^{14,15}$, Peter Lichter ${ }^{10}$, Bernhard Radlwimmer ${ }^{10}$, Michael Platten $^{1,8}$, Stefan M Pfister ${ }^{5,9}$, Andreas von Deimling ${ }^{2,7}$, Michael Weller ${ }^{16}$, Wolfgang Wick ${ }^{1,6}$ *These authors contributed equally to this manuscript

${ }^{1}$ Departments of Neurooncology, ${ }^{2}$ Neuropathology, ${ }^{3}$ Neurosurgery, ${ }^{4}$ Neuroradiology and ${ }^{5}$ Pediatric Oncology, Hematology, and Immunology, Heidelberg University Hospital and German Cancer Consortium (DKTK) and German Cancer Research Center (DKFZ), Clinical Cooperation Units ${ }^{6}$ Neurooncology, ${ }^{7}$ Neuropathology and ${ }^{8}$ Neuroimmunology and Brain Tumor Immunology, ${ }^{9}$ Division of Pediatric Neurooncology, ${ }^{10}$ Division of Molecular Genetics, ${ }^{11}$ Division of Biostatistics, 69120 Heidelberg; ${ }^{12}$ Department for Neuropathology, Institute of Pathology, Medical University of Hannover, 30625 Hannover; ${ }^{13}$ Department of Neuropathology, University of Bonn Medical Center, 53105 Bonn, all Germany; ${ }^{14}$ Department of Neuropathology, Heinrich-Heine-University and ${ }^{15}$ DKTK, partner site Essen/Düsseldorf, 40225 Düsseldorf, all Germany; ${ }^{16}$ Department of Neurology, University Hospital Zurich, Frauenklinikstrasse 26, CH-8091 Zurich, Switzerland 


\section{Supplementary Methods}

\section{Patients and tumor samples}

The NOA-04 trial (NCT00717210) for patients with newly diagnosed anaplastic gliomas compared the efficacy and safety of initial radiotherapy, followed by chemotherapy (temozolomide or procarbazine, lomustine and vincristine) at progression or occurrence of unacceptable toxicity with the inverse sequence in patients with newly diagnosed anaplastic gliomas. In this trial, both sequences achieved similar results [9]. Median follow-up time was 54 months. All patients consented to exploratory molecular analyses performed with study data and materials. The original phase III trial was approved by the Ethics Committee (EC) at the University of Tuebingen, Germany, and subsequently all local ECs of the participating clinical centers.

All tumors were banked at the time of primary diagnosis between 1994 and 2013 in accordance with research ethics board approval from the respective institutes. Informed consent was obtained from all patients included in this study. All samples were independently reviewed by neuropathologists (T.P. (NOA-04), A.v.D. and A.K.) according to the WHO guidelines. The study additionally included data from 55 glioblastoma samples reported before [8].

\section{Preprocessing of the 450k Infinium methylation data}

The raw methylation data collected from different sources was preprocessed together by using the function preprocessIllumina of the Bioconductor Software package Minfi [1]. This function is an open source implementation of the normalization procedure developed by Illumina which is also available in Illumina's GenomeStudio Software. In brief, this procedure uses different internal control probes located on the array to perform background subtraction and normalization of the two measured intensity channels. 


\section{Unsupervised clustering of methylation data}

For unsupervised hierarchical clustering we selected the 10,000 most variable methylated probes that showed highest median absolute deviation (MAD) across beta values. Pairwise similarity between cases was calculated using euclidean distance and hierarchical clustering was performed using Ward's linkage method. To cluster $\mathrm{CpG}$ probes we applied hierarchical clustering using 1-centered Pearson correlation as similarity measure and average linkage as hierarchical clustering method. The same procedure was applied to all three data sets. As indicated by the volcano plot in Figure 1 of the Supplementary Material, the CIMP status is the dominating signal that might overlay other structures in the data, i.e., unknown molecular subgroups. Therefore, to select probes not affected by the CIMP signal and reveal such underlying subgroups, unspecific variance filtering and clustering was applied in the same way as described above to the two subgroups CIMP $^{\text {pos }}$ and CIMP ${ }^{\text {neg }}$.

\section{Detection of copy-number aberrations}

Copy-number data were computed from the 450k Infinium methylation array as described previously [8]. Low-resolution copy number variations were detected from the 450k Infinium methylation array in a custom approach using the sum of both methylated and unmethylated signals. Probes found to be highly variant in the six normal cerebellum samples were excluded from the analysis according to the following criteria: Removal of probes not within the 0.05 and 0.85 quantile of median summed values or over the 0.8 quantile of the median absolute deviation. Log-ratios of samples to the median value of control samples were calculated, and sample noisiness was determined as the median absolute deviation of adjacent probes. Probes were then combined by joining 20 adjacent probes, and resulting genomic windows less than $100 \mathrm{~kb}$ in size were iteratively merged with adjacent windows of smaller size. Windows of more than $5 \mathrm{Mb}$ were excluded from analysis, resulting in a total of 8,654 
windows throughout the genome. For each window, the median probe value was calculated and shifted to minimize the median absolute deviation from all windows to zero for every sample. Segmentation was performed by applying the circular binary algorithm using the following settings: min. width $=10$, nperm $=32000$, alpha $=0.001$, undo.splits="sdundo", undo.SD=2.2 [7]. The median value of windows contained in each segment was calculated, and classified as homozygous or hemizygous deletion, neutral, gain or high-level amplification by the following arbitrary thresholds: $-0.96,-0.24,0.12$ and 0.72 .

The resulting copy-number plots were individually curated for $1 \mathrm{p} / 19 \mathrm{q}$ codeletion, EGFR amplification, homozygous $C D K N 2 A$ deletion and copy-number changes affecting chromosomes 7 and 10 (examples in Supplementary Figure 6). 


\section{Supplementary Discussion}

Methylation of the $M G M T$ promoter is a well-established biomarker predicting benefit from temozolomide in glioblastomas [5], which has recently been prospectively confirmed in the RTOG-0525 trial [4]. In anaplastic gliomas however, the situation is more complex, as the prognostic role of $M G M T$ promoter methylation has been shown to be independent of treatment in two phase III trials [3, 9]. A recent analysis suggested an interdependence between $I D H$ status and $M G M T$ methylation. In $I D H$ wild type tumors, $M G M T$ is predictive specifically for benefit from alkylating chemotherapy, while it is prognostic in IDH mutant tumors [10]. The molecular concordance between CIMP $^{\text {neg }}$ (IDH wild type) anaplastic gliomas and glioblastomas, supports the similar relevance of $M G M T$ in $I D H$ wild type anaplastic gliomas and glioblastomas (Supplementary Figure 3). In CIMP ${ }^{\text {pos }}$ tumors on the other hand, the MGMT-STP27 algorithm, a logistic regression model based on the methylation in two CpGs in the differentially methylated regions 1 and 2 of MGMT [2], indicated a methylated MGMT promoter in 182 of 191 tumors. Of note however, in our database at the DKFZ which comprises methylation data of more than 4000 brain tumors covering most known entities we observed consistent batch effects between samples coming from FFPE and fresh frozen material for specific CpG probes. Probe cg12434587 which is one of the two probes involved in the MGMT-STP27 model seems to be affected by this batch effect. Our current estimate for the batch effect for probe cg12434587 is a $\log 2$ difference around 2 for the methylated fluorescence channel. Such an effect may result in methylation difference of up 0.3 on the beta scale. Therefore, as the MGMT-STP27 was trained on GBM samples from fresh frozen material its ability to correctly predict the MGMT status of samples coming from FFPE material might be limited. On the other hand, this high rate of $M G M T$ promoter methylation has been reported before in $I D H$ mutant gliomas [6]. Interestingly, the $9 M G M T$ unmethylated tumors all belonged to the CIMP-non-codel 
group, an observation which is further corroborated when considering the results of the centrally performed methylation-specific PCR (MSP) in NOA-04: per MSP, 38 / 42 (90\%) CIMP-codel tumors, but only 24 / 46 (52\%) of CIMP-non-codel tumors had a methylated MGMT promoter (Fisher's exact test, $\mathrm{p}<0.001$ ). Mulholland et al. suggested that the discrepancy between their finding of nearly $100 \%$ MGMT promoter methylation and the MSP results might be due to low methylation especially in the MSP-forward region, while methylation is higher in surrounding CpGs, still leading to reduced gene expression [6]. Hence, it seems possible that the prognostic effect seen with MSP might rather reflect a separation between CIMP-codel tumors (which are almost always methylated per MSP) and some CIMP-non-codel tumors. 
Supplementary Figure 1. Volcano plot for differentially methylated probes (using median difference and Wilcoxon's rank sum test). Probes with a significantly $(\mathrm{p}<0.05)$ different median methylation between both groups after false discovery rate correction are shown in red.

Supplementary Figure 2. Overall survival by CIMP status in anaplastic gliomas.

Supplementary Figure 3. Progression-free survival by $M G M T$ status and treatment arm (radio- vs. chemotherapy) in the CIMP ${ }^{\text {neg }}$ NOA-04 samples $(\mathrm{n}=24)$.

Supplementary Figure 4. Prediction error curves for time to treatment failure in Cox regression models including histology only, molecular classification only, or both.

Supplementary Figure 5. Overall survival by WHO grade in CIMP ${ }^{\text {neg }}$ tumors.

Supplementary Figure 6. Copy-number plots examples for a case with 1p/19q codeletion (a) and another case with EGFR amplification, CDKN2A deletion, gain of chromosome 7 and loss of chromosome 10 (b). 
Supplementary Table 1. Baseline patient characteristics of the NOA-04 cohort

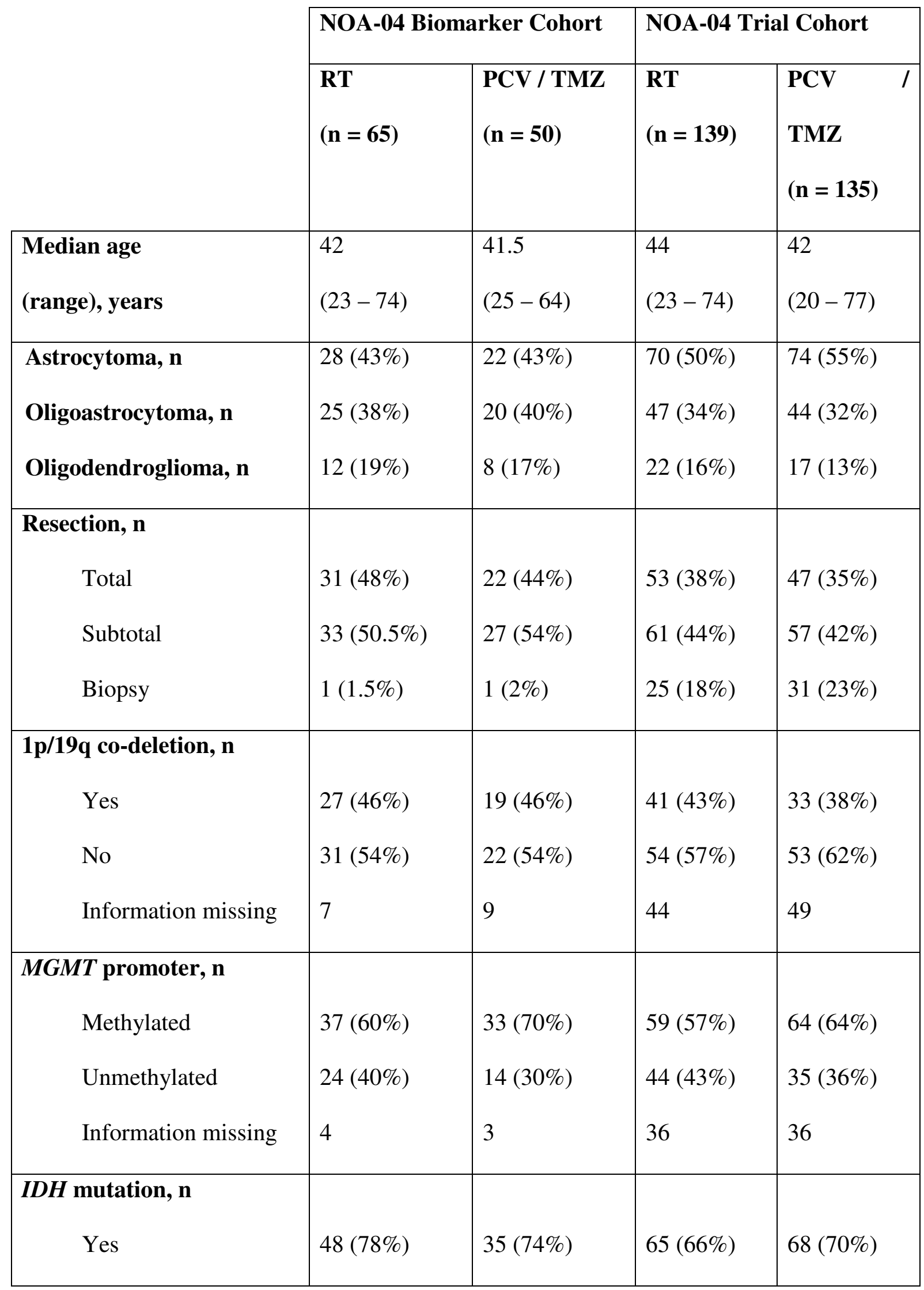


ANEU-D-14-00273R SUPPLEMENT -9-

\begin{tabular}{|l|l|l|l|l|}
\hline No & $13(22 \%)$ & $12(26 \%)$ & $33(34 \%)$ & $29(30 \%)$ \\
Information missing & 4 & 3 & 41 & 38 \\
\hline
\end{tabular}

RT, radiotherapy; PCV, procarbazine, CCNU \& vincristine; TMZ, temozolomide 
Supplementary Table 2. Cox regression for TTF by reference histology in NOA-04

\begin{tabular}{|l|l|l|l|}
\hline Variable & HR & 95\% CI & p \\
\hline AO vs. AA & 0.64 & $0.29-1.38$ & 0.257 \\
\hline AOA vs. AA & 0.39 & $0.2-0.77$ & 0.006 \\
\hline
\end{tabular}

AA, anaplastic astrocytoma; AO, anaplastic oligodendrogliomas; AOA, anaplastic oligoastrocytoma 


\section{Supplementary References}

1. Aryee MJ, Jaffe AE, Corrada-Bravo H, Ladd-Acosta C, Feinberg AP, et al. (2014) Minfi: a flexible and comprehensive Bioconductor package for the analysis of Infinium DNA methylation microarrays. Bioinformatics 30:1363-1369

2. Bady P, Sciuscio D, Diserens A-C, Bloch J, van den Bent MJ, et al. (2012) MGMT methylation analysis of glioblastoma on the Infinium methylation BeadChip identifies two distinct $\mathrm{CpG}$ regions associated with gene silencing and outcome, yielding a prediction model for comparisons across datasets, tumor grades, and CIMP-status. Acta Neuropathol. 124:547-560

3. Van den Bent MJ, Dubbink HJ, Sanson M, van der Lee-Haarloo CR, Hegi M, et al. (2009) MGMT promoter methylation is prognostic but not predictive for outcome to adjuvant PCV chemotherapy in anaplastic oligodendroglial tumors: a report from EORTC Brain Tumor Group Study 26951. J. Clin. Oncol. 27:5881-5886

4. Gilbert MR, Wang M, Aldape KD, Stupp R, Hegi ME, et al. (2013) Dose-Dense Temozolomide for Newly Diagnosed Glioblastoma: A Randomized Phase III Clinical Trial. J. Clin. Oncol. 31:4085-4091

5. Hegi ME, Diserens A-C, Gorlia T, Hamou M-F, de Tribolet N, et al. (2005) MGMT gene silencing and benefit from temozolomide in glioblastoma. N. Engl. J. Med. 352:997-1003

6. Mulholland S, Pearson DM, Hamoudi RA, Malley DS, Smith CM, et al. (2012) MGMT CpG island is invariably methylated in adult astrocytic and oligodendroglial tumors with IDH1 or IDH2 mutations. Int. J. Cancer 131:1104-1113

7. Olshen AB, Venkatraman ES, Lucito R \& Wigler M (2004) Circular binary segmentation for the analysis of array-based DNA copy number data. Biostatistics $5: 557-572$

8. Sturm D, Witt H, Hovestadt V, Khuong-Quang D-A, Jones DTW, et al. (2012) Hotspot mutations in $\mathrm{H} 3 \mathrm{~F} 3 \mathrm{~A}$ and IDH1 define distinct epigenetic and biological subgroups of glioblastoma. Cancer Cell 22:425-437

9. Wick W, Hartmann C, Engel C, Stoffels M, Felsberg J, et al. (2009) NOA-04 randomized phase III trial of sequential radiochemotherapy of anaplastic glioma with procarbazine, lomustine, and vincristine or temozolomide. J. Clin. Oncol. 27:58745880

10. Wick W, Meisner C, Hentschel B, Platten M, Schilling A, et al. (2013) Prognostic or predictive value of MGMT promoter methylation in gliomas depends on IDH1 mutation. Neurology 81:1515-1522 


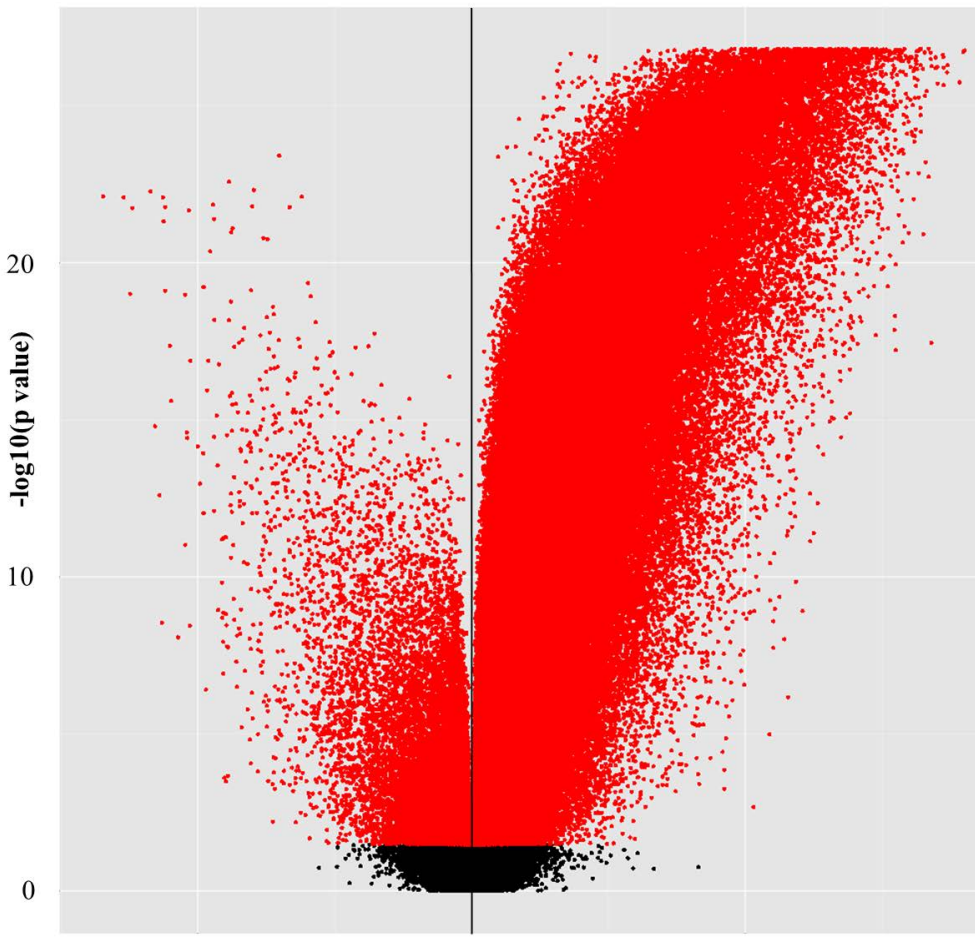

Higher median methylation in 


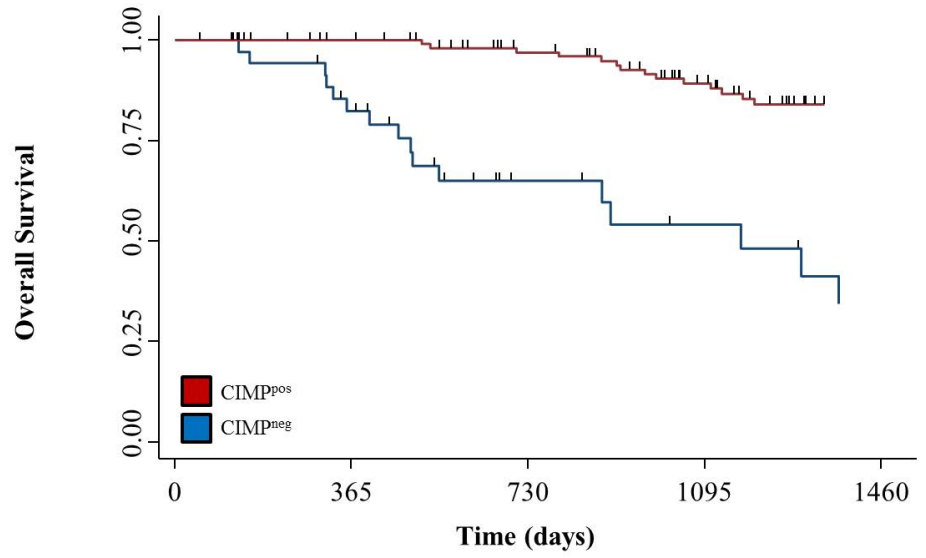

Number at risk

CIMP $^{\text {neg }} 37$

20

10

4

CIMPpos 120

105

80

47 


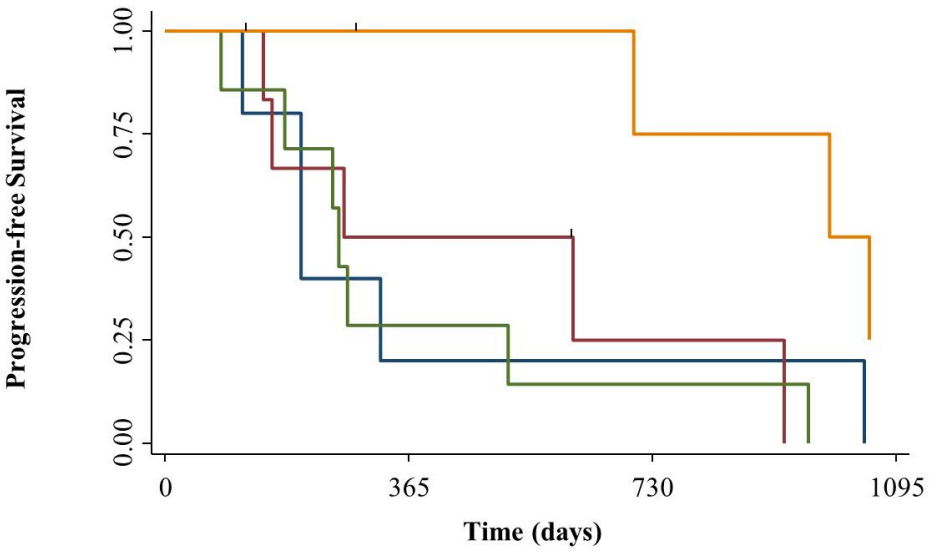

Radiotherapy / MGMT unmeth $(\mathrm{n}=5)$

Radiotherapy / MGMT meth $(n=7)$

Chemotherapy / MGMT unmeth $(n=7)$

Chemotherapy / MGMT meth $(n=5)$ 


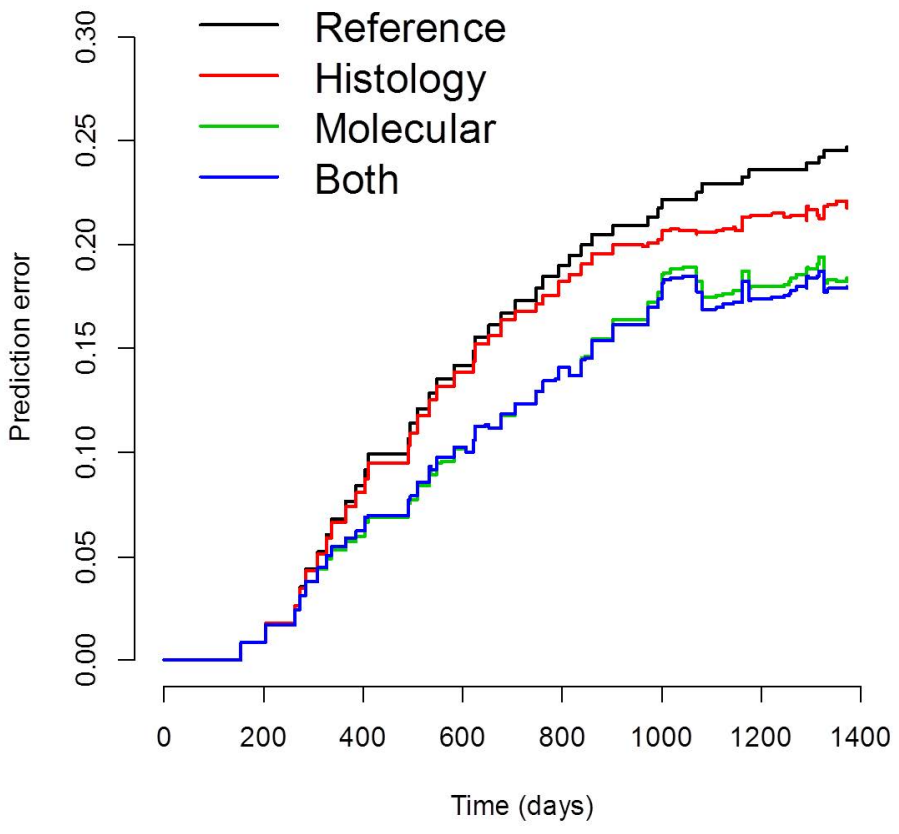




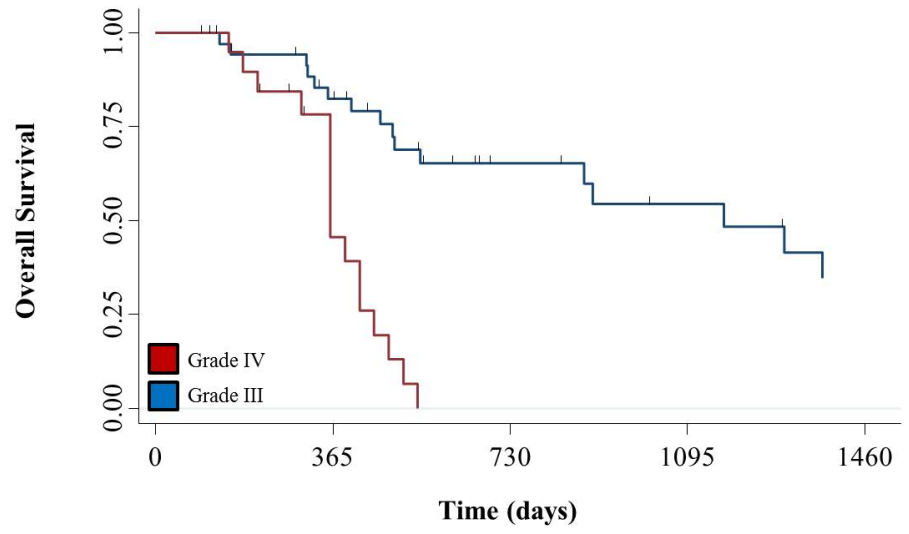

Number at risk

Grade III 37

Grade IV 22 
a)

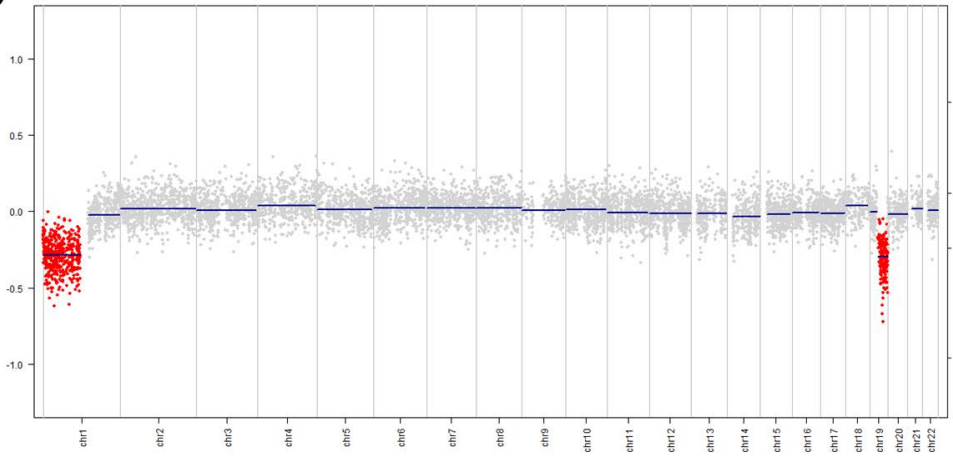

b)

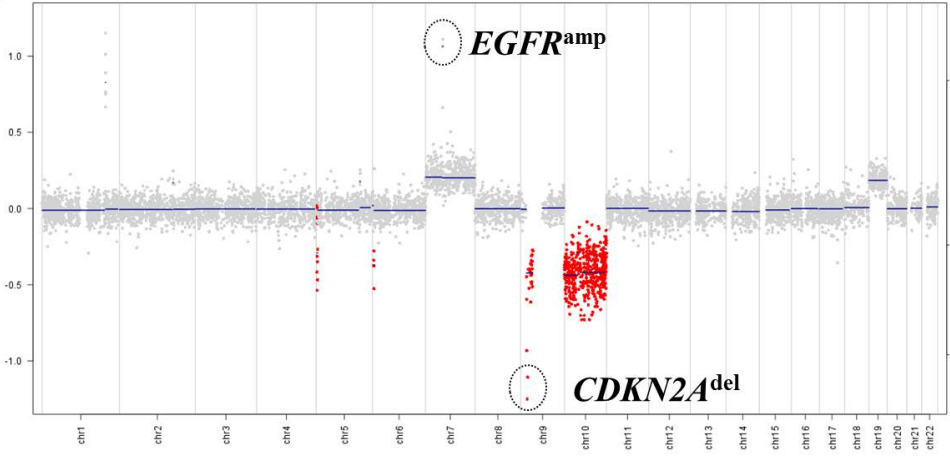

